\title{
Simulations of atomic-scale sliding friction
}

Sørensen, Mads Reinholdt; Jacobsen, Karsten Wedel; Stoltze, Per

Published in:

Physical Review B

Link to article, DOI:

10.1103/PhysRevB.53.2101

Publication date:

1996

Document Version

Publisher's PDF, also known as Version of record

Link back to DTU Orbit

Citation (APA):

Sørensen, M. R., Jacobsen, K. W., \& Stoltze, P. (1996). Simulations of atomic-scale sliding friction. Physical Review B, 53(4), 2101-2113. https://doi.org/10.1103/PhysRevB.53.2101

\section{General rights}

Copyright and moral rights for the publications made accessible in the public portal are retained by the authors and/or other copyright owners and it is a condition of accessing publications that users recognise and abide by the legal requirements associated with these rights.

- Users may download and print one copy of any publication from the public portal for the purpose of private study or research.

- You may not further distribute the material or use it for any profit-making activity or commercial gain

- You may freely distribute the URL identifying the publication in the public portal

If you believe that this document breaches copyright please contact us providing details, and we will remove access to the work immediately and investigate your claim. 


\title{
Simulations of atomic-scale sliding friction
}

\author{
M. R. Sørensen, K. W. Jacobsen, and P. Stoltze \\ Center for Atomic-Scale Materials Physics, Department of Physics, Technical University of Denmark, DK-2800 Lyngby, Denmark
}

(Received 12 June 1995)

\begin{abstract}
Simulation studies of atomic-scale sliding friction have been performed for a number of tip-surface and surface-surface contacts consisting of copper atoms. Both geometrically very simple tip-surface structures and more realistic interface necks formed by simulated annealing have been studied. Kinetic friction is observed to be caused by atomic-scale stick and slip which occurs by nucleation and subsequent motion of dislocations preferably between close-packed $\{111\}$ planes. Stick and slip seems to occur in different situations. For single crystalline contacts without grain boundaries at the interface the stick and slip process is clearly observed for a large number of contact areas, normal loads, and sliding velocities. If the tip and substrate crystal orientations are different so that a mismatch exists in the interface, the stick and slip process is more fragile. It is then caused by local pinning of atoms near the boundary of the interface and is therefore more easily observed for smaller contacts. Depending on crystal orientation and load, frictional wear can also be seen in the simulations. In particular, for the annealed interface necks which model contacts created by scanning tunneling microscope/ atomic force microscope tip indentations the sliding process involves breaking contacts leaving tip material behind on the substrate.
\end{abstract}

\section{INTRODUCTION}

The scientific interest in sliding friction and other tribological phenomena has existed for centuries. ${ }^{1-3}$ However, many unsolved questions still remain. Especially, an understanding of friction at the microscopic or at the atomic level is lacking. Within the past decade, there has been an increasing interest in the microscopic mechanisms of tribology and a research field called nanotribology has emerged. This has been motivated by the appearance of experimental techniques, such as the atomic force microscope (AFM). ${ }^{4}$ Furthermore, the rapid development in computer technology has provided the means to do large-scale calculations and simulations.

The AFM technique has made it possible in a number of experiments to obtain information about the friction behavior at the atomic level. Using an AFM with a tungsten tip on a graphite surface, atomic-scale modulations in the friction force were measured by Mate et al. ${ }^{5}$ Furthermore, atomicscale stick and slip was observed. Similar results have been reported on mica surfaces, ${ }^{6-8}$ diamond surfaces, ${ }^{9}$ and $\mathrm{MoS}_{2}$ surfaces. $^{10}$ The sliding of a Ir tip on a $\mathrm{Au}(111)$ film have been studied in ultrahigh vacuum (UHV), but atomicscale features could not be measured in this case. ${ }^{11}$

Information about the mechanical behavior of nanosize metallic contacts has been obtained by indentation experiments with a STM operating at room temperature ${ }^{12-16}$ or at 4 $\mathrm{K} .^{17,18}$ Measurements of the conductance provide information of the size and geometry of the contact. Similar measurements have been performed with a mechanically controllable break junction technique at $4 \mathrm{~K}$ or below. ${ }^{19}$

Ultimately, a combination of a STM and an AFM will make up a very valuable tool for nanotribological experiments. Recently, Agrait et al. have measured both the conductance and the adhesive force simultaneously for nanosize Au connective necks with a combined scanning force and tunneling microscope at $4 \mathrm{~K} .{ }^{20}$ Forces in the $\mathrm{nN}$ range were measured, and atomic-scale modulations in force and conductance were observed under tension and compression. Schaefer et al. also found force modulations in the $\mathrm{nN}$ range in AFM experiment on nanosize Au clusters. ${ }^{21}$

Finally, regarding friction at the atomic level, Hirano et al. have measured anisotropy in the friction force between two mica surfaces. ${ }^{22}$ The authors found that the friction force depends on the misorientation angle of the contacting surfaces. The friction decreases when the lattice mismatch between the surfaces increases.

On the theoretical side, there has been a number of studies of sliding friction between ideal atomically flat solid surfaces. McClelland has clarified the subject by a discussion of the independent oscillator model, ${ }^{23}$ which is based on the work by Tomlinson. ${ }^{24}$ Another model which has been used in this context is the Frenkel-Kontorova model. ${ }^{25,26}$ These simple one-dimensional models of infinitely large surfaces indicate that the frictional properties depend crucially on concepts, such as commensurability and interfacial bond strength. The models suggest that two ideal atomically flat surfaces, which are incommensurate and weakly interacting, can slide without any friction. This is called superlubricity. ${ }^{27}$ In the case of strongly interacting surfaces, nonadiabatic motion can occur, and this gives rise to kinetic friction.

Hirano and Shinjo have analyzed in details a more general model of quasistatic sliding friction in three dimensions. ${ }^{28}$ Qualitatively, the conclusions agree with those of the simpler models. However, based on numerical calculations for $\alpha$ iron, the authors conclude that nonadiabatic motion is unlikely to occur even in metallic systems. In a subsequent publication, they stress that in high dimensional systems, superlubricity can appear even for strongly interacting surfaces. $^{27}$

Sliding friction at the atomic level has been studied by molecular dynamics (MD) simulations by several authors. Landman and co-workers have found atomic-scale stick and slip behavior when shearing a $\mathrm{Si}$ tip on a $\mathrm{Si}(111)$ surface $^{29}$ 


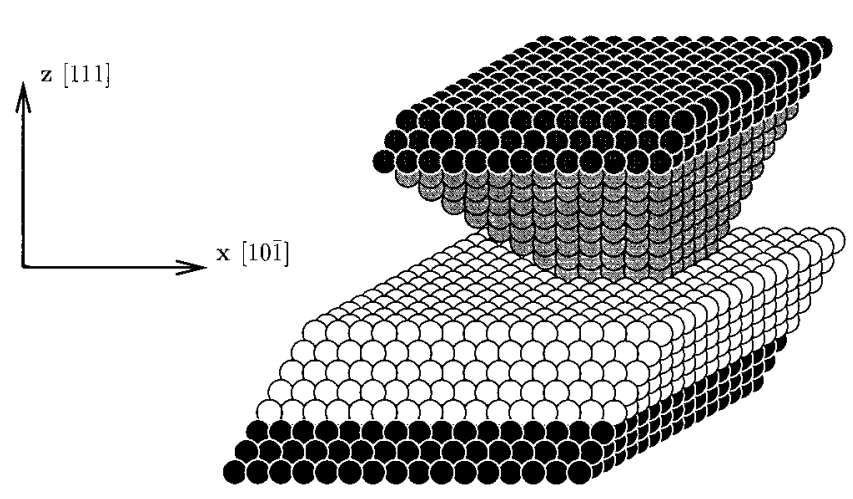

FIG. 1. A flat $\mathrm{Cu}(111)$ tip in matching contact with a $\mathrm{Cu}(111)$ substrate. There are $5 \times 5$ atoms in the bottom layer of the tip.

and $\mathrm{a} \mathrm{CaF}_{2}$ tip on a $\mathrm{CaF}_{2}$ substrate. ${ }^{30}$ Stick and slip was also observed by Harrison et al. in the case of sliding between two hydrogen-terminated diamond (111) surfaces, ${ }^{31}$ and by Nieminen et al. for a $\mathrm{Cu}$ tip on a $\mathrm{Cu}(100)$ surface. $^{32}$ In some of these studies, wearless friction occurs. ${ }^{31}$ In others, wear takes place during the sliding. ${ }^{29,30,32}$

Computer simulation has proven to be a valuable tool for interpreting experimental results and for suggesting new experiments. ${ }^{12-14}$ Furthermore, simulations provide a testing ground for simpler approximate theories. In this way, simulations can help to establish the connection between experiments and simpler models.

The mentioned experimental possibilities form the key motivation for the present paper. In the paper, we describe a number of simulation studies of atomic-scale sliding friction in different kinds of tip-surface and surface-surface contacts. In all simulations, the material is copper. For direct quantitative comparison with experiments, the possibilities of studying nanosize metallic contacts in UHV are most encouraging. In the simulations, the dependence of parameters such as normal load, contact area, sliding velocity, and temperature is investigated. Furthermore, the effect of the interfacial mismatch is studied. The simulations give insight into the frictional behavior observed in AFM experiments, e.g., atomic-scale stick and slip.

The paper is organized as follows. Section II contains a description of the simulation methods. In Sec. III, we present and discuss the results of the simulations. Finally, in Sec. IV, we conclude.

\section{METHODS}

An example of a system that is studied in this work is shown in Fig. 1. A flat tip of $\mathrm{Cu}$ atoms is placed on a $\mathrm{Cu}(111)$ substrate. The tip consists of $\mathrm{Cu}(111)$ layers stacked upon each other. There are $5 \times 5$ atoms in the bottom layer of the tip and this corresponds to a contact area of approximately $140 \AA^{2}$. Periodic boundary conditions are used in the two dimensions parallel to the surface plane. In the third dimension, fixed boundary conditions are established by static atoms in the three bottom layers of the substrate and in the three top layers of the tip. The distances between the static atoms are kept fixed at the bulk values. There are five and eight dynamic layers in the substrate and in the tip, respectively. The total number of atoms is 3175 .
The interatomic potentials are derived from the effective medium theory (EMT). ${ }^{33,34}$ The EMT provides an approximate and computationally efficient description of the essential physics of the interatomic interactions in simple metallic systems. The EMT has been applied in studies of, e.g., diffusion, surface reconstructions, surface premelting, surface defects and clusters. ${ }^{34,35}$ The EMT has also been used in simulations of dislocations and grain boundaries, ${ }^{36,37}$ and atomic-size point contacts. $^{12,13}$

Two kinds of simulations have been performed. In the first method, the static atoms in the top of the tip are displaced in the sliding direction in steps of $0.05 \AA$, and the positions of the dynamic atoms are relaxed by a local energy minimization procedure similar to steepest descent. ${ }^{38}$ To get good accuracy, the steps are as small as $0.005 \AA$ near critical positions. This method corresponds to sliding at the temperature $T=0 \mathrm{~K}$ in the quasistatic limit $(v \rightarrow 0)$, and it has the advantage of being very well defined. In the following, the minimization procedure is called molecular dynamics minimization.

The second method is based on molecular dynamics (MD) at a finite temperature $T>0$. In these simulations, the static atoms are displaced in the sliding direction before each time step. The temperature is controlled by imposing fluctuating forces and frictional forces on the atoms in one layer of the substrate and in one layer of the tip. (These two layers are the ones closest to the static atoms.) In other words, the dynamics of these atoms are controlled by a Langevin equation. The other dynamic atoms obey a usual Newtonian equation of motion. The numerical integration of the equations of motion is done by using a velocity Verlet algorithm. The length of a time step in the integration algorithm is $\Delta t=5.4 \times 10^{-15} \mathrm{~s}$. The distances between the static atoms are chosen according to the thermal expansion in bulk at the relevant temperature.

\section{RESULTS AND DISCUSSION}

\section{A. $\mathrm{Cu}(111)$ tip/ $\mathrm{Cu}(111)$ surface, matching surfaces}

In this section, the first results of the simulations are presented. The system used in the simulations is the one shown in Fig. 1. The tip is sliding in the close-packed [ 101$]$ direction on the substrate (to the left in Fig. 1). A coordinate system is chosen with the $x$ axis in the [101] direction (opposite to the direction of motion). The $z$ axis is along the [111] direction, perpendicular to the surface of the substrate, directed away from the substrate.

Figure 2 shows the three components of the force on the tip as a function of the sliding distance for a simulation at $T=0 \mathrm{~K}$. The total sliding distance is a little longer than two periods of the surface of the substrate. The lateral force $F_{x}$ has a sawtooth shape, indicating atomic-scale stick and slip motion. The motion can be described as follows. To begin with, the tip sticks to the substrate and the tip bends elastically as the top of the tip is displaced. This builds up a force on the tip directed opposite to the direction of motion. Suddenly the tip slips on the substrate, and the force drops significantly. Then the tip sticks again, a force is built up, the tip slips, and the force drops. This is repeated periodically with a periodicity of $2.6 \AA$, which equals the distance between 


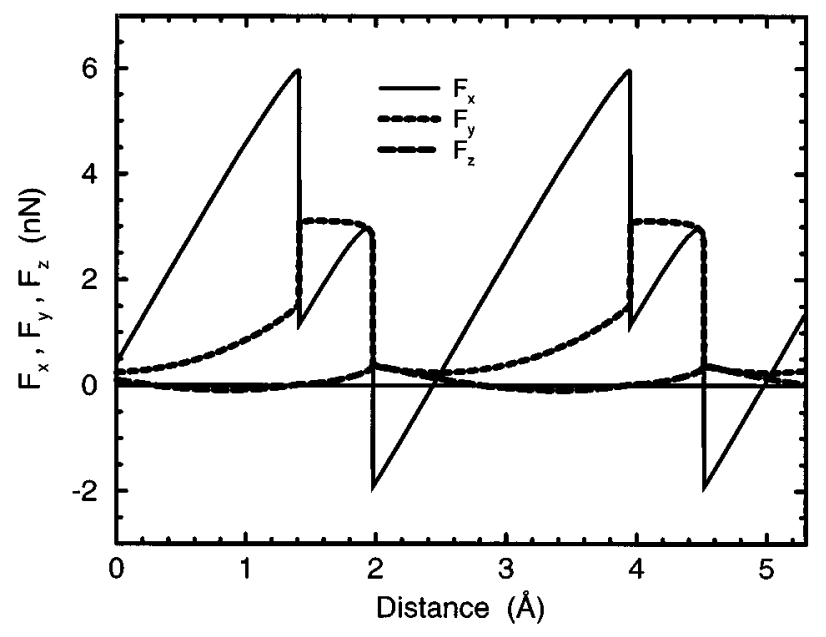

FIG. 2. The three components of the force on the tip as a function of the sliding distance. The contacting $\mathrm{Cu}(111)$ surfaces have identical orientation. $T=0 \mathrm{~K}$.

nearest-neighboring atoms in the $[\overline{1} 01]$ direction of the substrate. In each period, two slips take place.

The behavior described above is due to the hexagonal structure of the surface of the substrate. While the atoms in the top of the tip are displaced along a straight line, the atoms in the bottom layer of the tip jump discontinuously (nonadiabatically) in a zigzag pattern from fcc to hcp positions on the $\mathrm{Cu}(111)$ surface. When the atoms jump from fcc to hcp positions, the tip deforms substantially in the $y$ direction, and mechanical stresses in this direction are built up. When the atoms move back to the next fcc positions, the mechanical stresses in the $y$ direction are released. This is the reason why there is a large "sawtooth" and a small "sawtooth" in the $F_{x}$ curve in Fig. 2. The variations in the $F_{y}$ curve is understood in the same way. The $F_{z}$ curve is rather flat compared to the $F_{x}$ and $F_{y}$ curves. The observed atomic-scale stick and slip may also be called "ratcheting" in order to distinguish it from macroscopic stick and slip. ${ }^{39}$ The former is due to the corrugation of the surface potential, while the latter arises from the difference between the static and kinetic friction coefficient.

We note that the zigzag motion described above is similar to the "two-dimensional stick and slip phenomenon" observed in AFM experiments with a $\mathrm{Si}_{3} \mathrm{~N}_{4}$ tip on (hexagonal) mica and $\mathrm{MoS}_{2}$ surfaces by Fujisawa and co-workers. ${ }^{8,10}$

It is evident from Fig. 2 that the average value $\left\langle F_{x}\right\rangle$ of the lateral force is positive, so there is, in fact, kinetic friction. The average vertical separation between the tip and the substrate is $2.03 \AA$, and it changes only within $0.02 \AA$ during a scan. This distance is equal to the distance between $\{111\}$ planes in the bulk within a few percent.

When changing the position of the tip in the $y$ direction before scanning in the $x$ direction, the size of the two "sawtooths" in the $F_{x}$ curve vary. Experimentally, this has been utilized for making two-dimensional images with atomic resolution of the surface topography by scanning in the $x$ direction at different $y$ positions and coloring according to the value of $F_{x} \cdot{ }^{5,7,8,10}$ In the simulations, it is mainly the relative sizes of the two "sawtooths" that changes, while $\left\langle F_{x}\right\rangle$ remains almost constant.

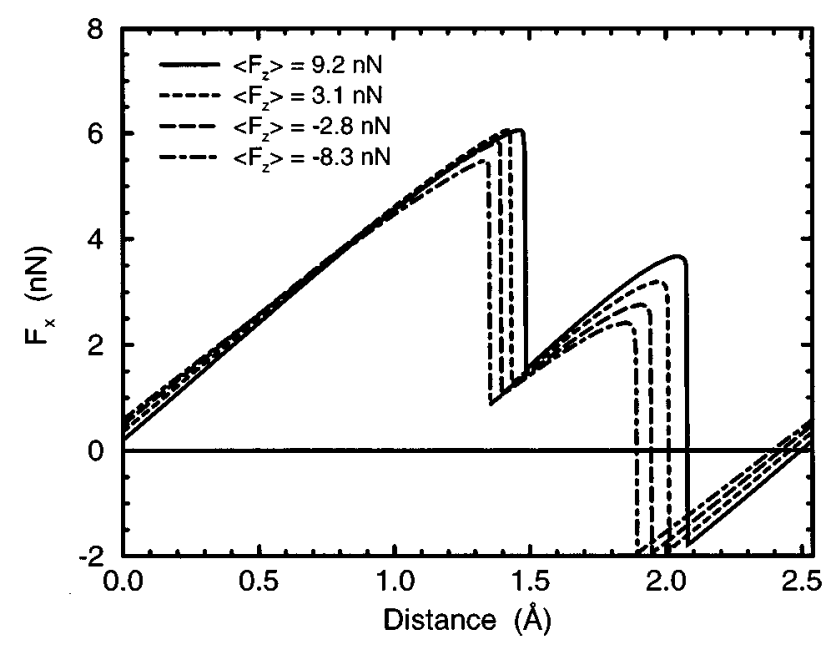

FIG. 3. The lateral force $F_{x}$ on the tip as a function of the sliding distance at different average loads $\left\langle F_{z}\right\rangle$. The contacting $\mathrm{Cu}(111)$ surfaces have identical orientation. $T=0 \mathrm{~K}$.

The number of layers in the tip and in the substrate also have an effect on $\left\langle F_{x}\right\rangle$. With fewer atomic layers, the system is elastically more stiff. The maximum values of $F_{x}$ (before the slips) are unchanged, but the values of $F_{x}$ right after the slip become smaller, so that $\left\langle F_{x}\right\rangle$ becomes smaller. In the following, the dependence of parameters such as temperature, load, contact area, and sliding velocity is studied.

\section{Effect of load}

The type of simulations described above has been repeated for a number of different heights of the static part of the tip. Each height corresponds to a certain average load $\left\langle F_{z}\right\rangle$. One period of some of the resulting $F_{x}$ curves are shown in Fig. 3. The trend is that for higher loads, the slips occur at a larger displacement, compared to simulations at lower loads. As a consequence of this, $\left\langle F_{x}\right\rangle$ and, in general, also the local maximum values $F_{x, \max 1}$ and $F_{x, \max 2}$ increase with the load. As illustrated in Fig. $4,\left\langle F_{x}\right\rangle$ increases approximately linearly as a function of $\left\langle F_{z}\right\rangle$ in a wide range, including both negative and positive loads. The slope of the curve is $\alpha \approx 0.03$.

In most cases of macroscopic friction, the friction force increases proportionally to the load. This relation is known as Amontons law. In the standard model by Bowden and Tabor, ${ }^{3}$ the contact area increases proportionally to the load in order to keep a constant normal pressure, which is basically the yield pressure of the material. By assuming a certain constant shear stress of the contact, Amontons law follows. For clean metals, the friction coefficient is high, i.e., higher than 1.

In the atomic-scale simulations, the situation is quite different from the one described above. In the simulations, the contact area is a constant and because of adhesion, the contact is established even at negative loads. So the data in Fig. 4 can be interpreted in the way that the average shear stress $\langle\tau\rangle$ at the interface increases linearly with a nonzero offset as a function of the average normal pressure $\left\langle p_{z}\right\rangle$. This can be written as

$$
\langle\tau\rangle=\tau_{0}+\alpha\left\langle p_{z}\right\rangle
$$




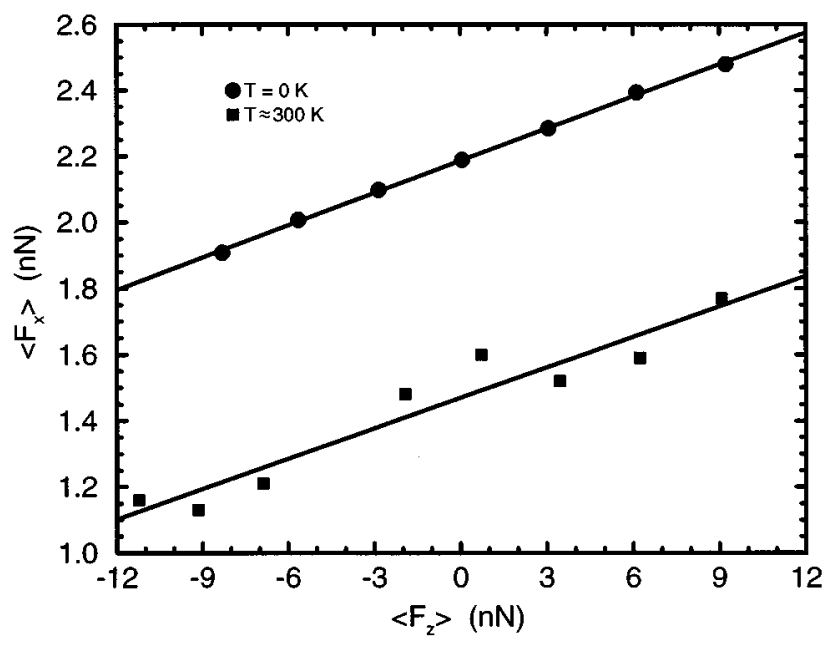

FIG. 4. The average lateral force $\left\langle F_{x}\right\rangle$ as a function of the average load $\left\langle F_{z}\right\rangle$. The contacting $\mathrm{Cu}(111)$ surfaces have identical orientation.

Figure 4 also presents results from MD simulations performed at the temperature $T \approx 300 \mathrm{~K}$, with a sliding velocity $v \approx 2 \mathrm{~m} / \mathrm{s}$. The general trend in these simulations is the same as in the simulations at $T=0 \mathrm{~K}$, but quantitatively one finds that at any load, the slips occur at a smaller tip displacement. Both the $F_{x, \max }$ and $\left\langle F_{x}\right\rangle$ are smaller at $300 \mathrm{~K}$ than at $0 \mathrm{~K}$. The best linear fit based on the square symbols in Fig. 4 give a slope of $\alpha \approx 0.03$. This indicates, that $\alpha$ in Eq. (1) is independent of temperature, while $\tau_{0}$ decreases with temperature. A relationship of the form of Eq. (1) has been found to hold in experiments of boundary lubrication with monolayers of fatty acids between molecularly smooth mica surfaces. ${ }^{39,40}$

\section{Critical shear stress}

It is generally assumed that slip between lattice planes in solids occurs when the resolved shear stress exceeds some critical value $\tau_{\max }$ (the critical shear stress). In order to investigate this question, the shear stresses in the two ideal slip directions (the $[\overline{2} 11]$ and $[\overline{1} \overline{1} 2]$ directions) have been calculated for each of the simulations at $T=0 \mathrm{~K}$. The expressions are

$$
\tau_{1}=\frac{F_{x} \cos 30^{\circ}-F_{y} \sin 30^{\circ}}{A}
$$

and

$$
\tau_{2}=\frac{F_{x} \cos 30^{\circ}+F_{y} \sin 30^{\circ}}{A}
$$

where $A$ is the contact area. The value of $\tau_{1}$ just before the first slip and the value of $\tau_{2}$ just before the second slip are referred to as $\tau_{1}^{*}$ and $\tau_{2}^{*}$, respectively. Figure 5 shows $\tau_{1}^{*}$ and $\tau_{2}^{*}$ as a function of the corresponding normal pressure $p_{z}=F_{z} / A$. One finds that $\tau_{1}^{*}$ and $\tau_{2}^{*}$ are fairly close to each other in all cases, compared to the total range of $\tau_{1}$ and $\tau_{2}$. However, $\tau_{1}^{*}$ and $\tau_{2}^{*}$ do differ from one another, and this is due to the asymmetry of the motion, as discussed previously.

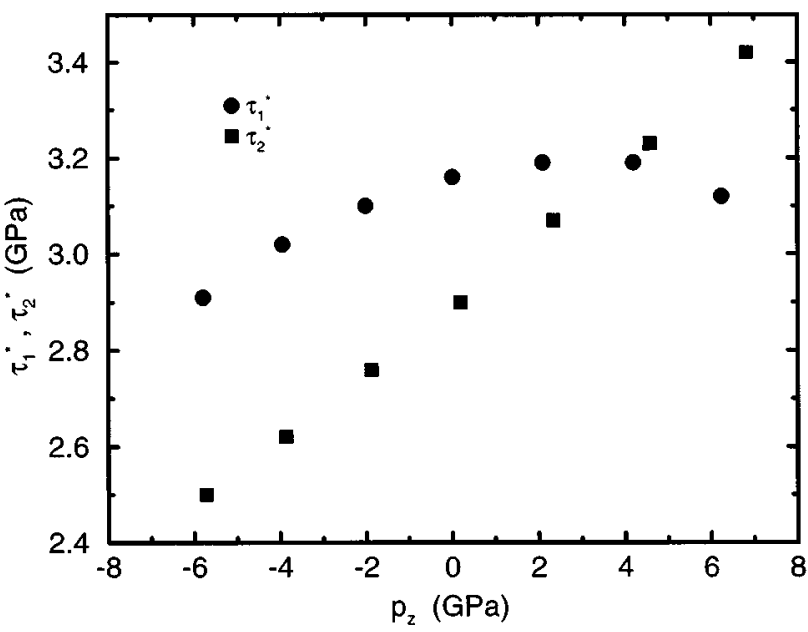

FIG. 5. The critical shear stresses $\tau_{1}^{*}$ and $\tau_{2}^{*}$ as a function of the corresponding normal pressure $p_{z}$. The contacting $\mathrm{Cu}(111)$ surfaces have identical orientation. $T=0 \mathrm{~K}$.

From Eq. (1), it seems reasonable to suggest that the critical shear stress should increase linearly with a nonzero offset as a function of the normal pressure. The $\tau_{2}^{*}$ points in Fig. 5 do fall closely around a straight line, but this is, however, not the case for the $\tau_{1}^{*}$ points.

On the one hand, Fig. 5 verifies that it is a reasonable first approximation to think in terms of ideal slips with a critical shear stress, but on the other hand, it clearly indicates that this ideal description is a simplification. The picture of ideal slips is least applicable at the first slip, where the elastic deformations and the atomic relaxations at the interface before the slip are rather large, especially at higher loads.

In the literature, there exists a number of different estimates of the theoretical shear stress for slip between $\{111\}$ planes in the fcc crystal structure. ${ }^{41}$ The estimates fall in the range from $\frac{1}{30} G$ to $\frac{1}{9} G$, where $G=3 c_{44}\left(c_{11}-c_{12}\right) /$ $\left[4 c_{44}+c_{11}-c_{12}\right]$ is the appropriate shear modulus. With the interatomic potentials used in the simulations, $G$ is equal to $38 \mathrm{GPa}$ at $T=0 \mathrm{~K}$. A typical critical shear stress $\tau_{\max }=3.0$ $\mathrm{GPa}$ in the simulations at $T=0 \mathrm{~K}$ then corresponds to $\frac{1}{13} G$, which falls inside the suggested range.

The increased atomic mobility at higher temperatures results in a lower critical shear stress. At $T \approx 300 \mathrm{~K}, \tau_{\max }$ approximately equals $2.3 \mathrm{GPa}$ at $p_{z}=0 \mathrm{GPa}$.

\section{Effect of contact area}

In the case of $\mathrm{Cu}(111)$ surfaces, the average friction force $\left\langle F_{x}\right\rangle$ is approximately proportional to the contact area $A$. This is illustrated in Fig. 6, which shows data from simulations with contact areas of $3 \times 3,5 \times 5,7 \times 7$, and $9 \times 9$ atoms. The resolved shear stresses $\tau_{1}$ and $\tau_{2}$ were also calculated in the four simulations, and the critical stresses $\tau_{1}^{*}$ and $\tau_{2}^{*}$ were the same as the ones in Sec. III A 2 within the computational accuracy.

\section{Mechanism of slip}

In this section, we examine in detail what happens during a slip event. A convenient way to get information of how the 


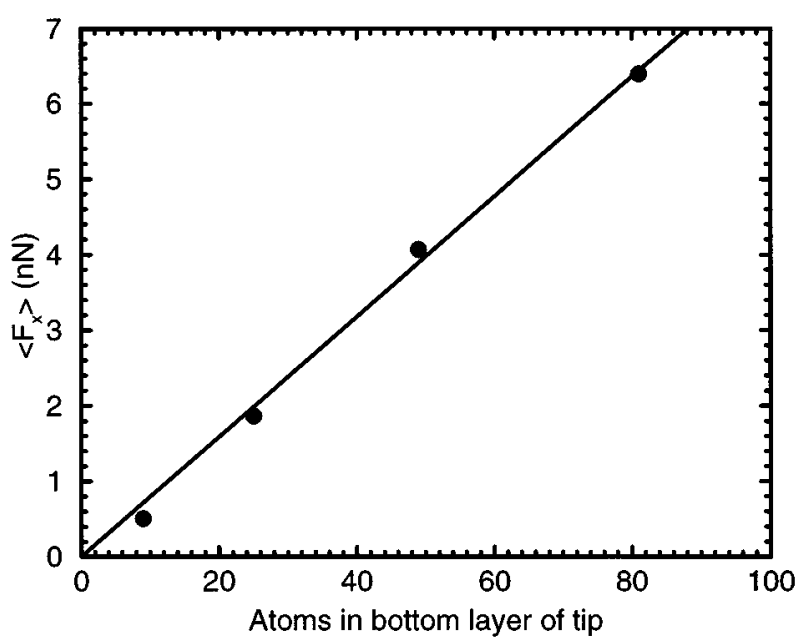

FIG. 6. The average lateral force $\left\langle F_{x}\right\rangle$ on the tip as a function of the sliding distance at four different contact areas. The straight line indicates the best linear fit through $(0,0)$. In these simulations, there are four and six dynamic layers in the substrate and in the tip, respectively. The height of the tip is the same in the simulations. The corresponding normal pressures are about $1 \mathrm{GPa}$. The contacting $\mathrm{Cu}(111)$ surfaces have identical orientation. $T=0 \mathrm{~K}$.

system evolves during a simulation is to record a number of snapshots of the atomic configurations. Since the slips occur very rapidly, it is necessary to record more pictures per unit of time than is usually the case. Figure 7 shows four such snapshots from a MD simulation of a system at $12 \mathrm{~K}$ with a sliding velocity of $2 \mathrm{~m} / \mathrm{s}$ and a contact area of $9 \times 9$ atoms. The time in the simulation between each snapshot is approximately $0.3 \mathrm{ps}$. The snapshots illustrate the motion of the atoms in the bottom layer of the tip during the slip event. The sliding direction is to the left in the figure.

Immediately before the slip, the positions of the atoms in the bottom layer of the substrate can be described as fcc positions, but the atoms are somewhat displaced in the sliding direction away from the ideal fcc positions. In picture (a)
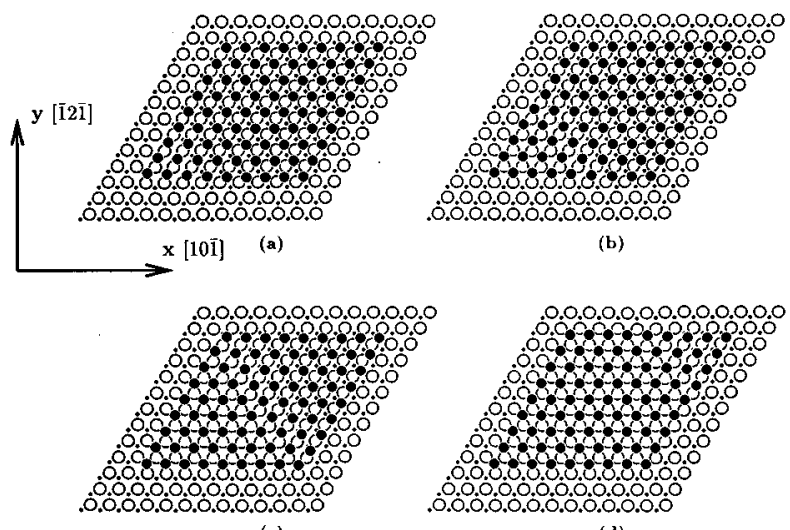

(c)

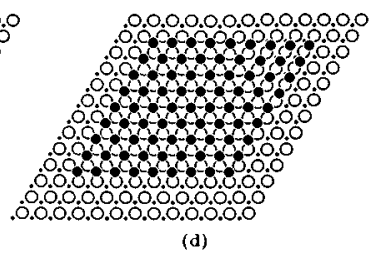

FIG. 7. Snapshots showing the $9 \times 9$ atoms in the bottom $\mathrm{Cu}$ (111) layer of the tip (black circles) and the atoms in the top $\mathrm{Cu}(111)$ layer of the substrate (white circles), during a slip event. The atoms in the second-topmost layer of the substrate are shown as black dots. These indicate the hcp positions on the surface of the substrate. The sliding direction is to the left. of Fig. 7, a few tip atoms in the lower-left corner have begun to move from fcc to hep positions. At this point, the slip is initiated. The slip proceeds as seen in the pictures (b)-(d), until the whole tip layer has slipped. From the snapshots, one finds that the slip occurs via a dislocation mechanism. In each picture, there is a slipped region and an unslipped region separated by a dislocation. The dislocation is nucleated at the corner and then it moves rapidly through the contact region. The dislocation can be described as a Shockley partial dislocation along the $[\overline{1} 10]$ direction with a Burgers vector $\frac{1}{6}[2 \overline{1} \overline{1}]$. Since the angle between the dislocation line and the Burgers vector is $30^{\circ}$, the dislocation has a mixed edge and screw character; predominantly screw.

The question is now why the slip is nucleated at the lower-left corner in Fig. 7. In Sec. III A 2, the slip initiation was discussed in terms of the shear stress in the slip direction, averaged over the contact region. However, the mechanical stress in the system is not distributed homogeneously. The local stress distribution can be calculated by assigning a stress tensor $\boldsymbol{\sigma}$ to each atom in the system. ${ }^{30}$ Figure 8 shows contour plots of the local stresses in a system just before a slip from fcc to hcp positions. The plots are based on snapshots from the simulation corresponding to Fig. 2. In the figure, three different stress quantities are plotted in two different cross sections of the system. The stress in (a) and (b) is the hydrostatic pressure $p$, in (c) and (d) it is $\sqrt{J_{2}}$, where $J_{2}$ is the second invariant of the stress deviator $\boldsymbol{\Gamma}$, and in (d) and (e) it is the shear stress in the direction of motion $-\tau_{x z} \cdot{ }^{42}$ The cross section in (a), (c), and (e) is parallel to the $x$ and $z$ axis (the [10ㅍ] and [111] directions), and the one in (b), (d), and (f) is parallel to the $z$ axis and an axis in the $[01 \overline{1}]$ direction. Both planes cut through the middle of the tip.

As it can be seen from Fig. 8, it is not simply the case that the shear stress in the direction of motion is largest at the nucleation center. One has to consider the state of stress more generally. From the plot of the hydrostatic pressure, one finds that, at the nucleation corner, there is a transition from tensile pressure (above the slip plane) to compressive pressure (below the slip plane). This could be of importance for the nucleation process. Furthermore, $\sqrt{J_{2}}$ is concentrated near the nucleation point.

For comparison, we have carried out simulations with a slab geometry in order to study the sliding of two infinite $\mathrm{Cu}(111)$ surfaces. Because of the periodic boundary conditions, there are no possible nucleation centers and the slips have to take place by moving all the atoms at an interface between two $\mathrm{Cu}(111)$ planes at the same time. This requires a significantly larger shear stress. The maximum lateral force per unit of area is in the order of $50 \%$ larger than for the tip-surface contact. Furthermore, the zigzag motion is not observed. The strain in the system after the first slip is so large that the second slip happens immediately after the first one.

\section{Effect of sliding velocity}

In this section, the effect of the sliding velocity is studied in MD simulations at $12 \mathrm{~K}$ and at $300 \mathrm{~K}$. The subject involves dynamical effects, such as excitations of phonons and energy dissipation. Because of the small time step in the 
(a)

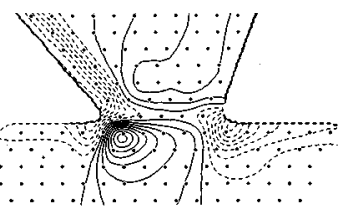

(c)
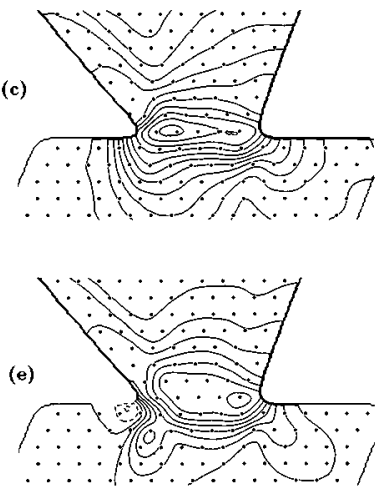

$\hat{1}^{z[111]} x^{x[10 \overline{1}]}$
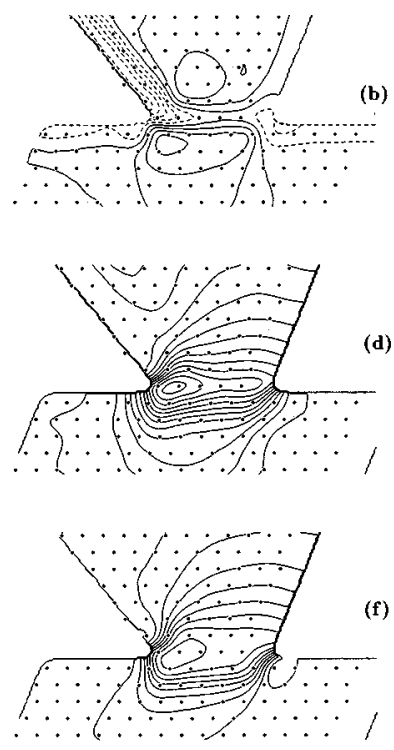

$\mathbf{z}^{\mathbf{z}[111]}$

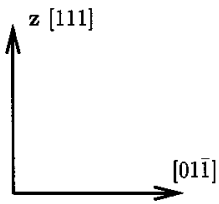

FIG. 8. Contour plots of the local state of stress for a $\mathrm{Cu}(111)$ tip on a $\mathrm{Cu}(111)$ substrate, just before the atoms in the bottom layer of the tip slip from fcc to hcp positions. The plots are based on snapshots from the simulation corresponding to Fig. 2. The contact area between the tip and the substrate consists of $5 \times 5$ atoms. The stresses are in (a) and (b) the hydrostatic pressure $p$, in (c) and (d) $\sqrt{J_{2}}$, where $J_{2}$ is the second invariant of the stress deviator $\boldsymbol{\Gamma}$, and in (e) and (f) the shear stress in the direction of motion $-\tau_{x z}$ (Ref. 42). The cross section in (a), (c), and (e) is parallel to the $x$ and $z$

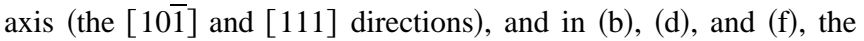
cross section is parallel to the $z$ axis and an axis in the [01 $\overline{1}]$ direction. Both planes cut through the middle of the tip. The difference between the stress at two neighboring contours is $0.5 \mathrm{GPa}$. Dashed lines indicate negative stresses. Contour plots of the resolved shear stress in the slip direction $\tau_{1}$ are similar to the plots of $-\tau_{x z}$.

integration algorithm and the limited computation time, it has only been possible to simulate sliding velocities higher than approximately $1 \mathrm{~m} / \mathrm{s}$. The temperature is controlled by applying frictional forces and fluctuating forces on the atoms in the top layer of the tip and in the bottom layer of the substrate. The average load $\left\langle F_{z}\right\rangle$ was about $0.2 \mathrm{nN}$, and the sliding velocities were in a range from $1 \mathrm{~m} / \mathrm{s}$ to $20 \mathrm{~m} / \mathrm{s}$. The results are shown in Fig. 9 and Fig. 10.

The curve in Fig. 9(a) shows the lateral force $F_{x}$ on the tip, as function of the sliding distance in a simulation at 300 $\mathrm{K}$, with a velocity of $2 \mathrm{~m} / \mathrm{s}$. The other two curves in Fig. 9(b) and Fig. 9(c) are from simulations at $12 \mathrm{~K}$ with velocities of $2 \mathrm{~m} / \mathrm{s}$ and $10 \mathrm{~m} / \mathrm{s}$, respectively. The curves are drawn from a number of data points that are connected by solid lines. Each data point has been calculated as the average over 100 time steps.

The fluctuations in the $F_{x}$ curve in Fig. 9(b) increase substantially immediately after each slip. During the subsequent stick, the fluctuations decay. The fluctuation patterns are almost identical in the last three periods. This indicates that the

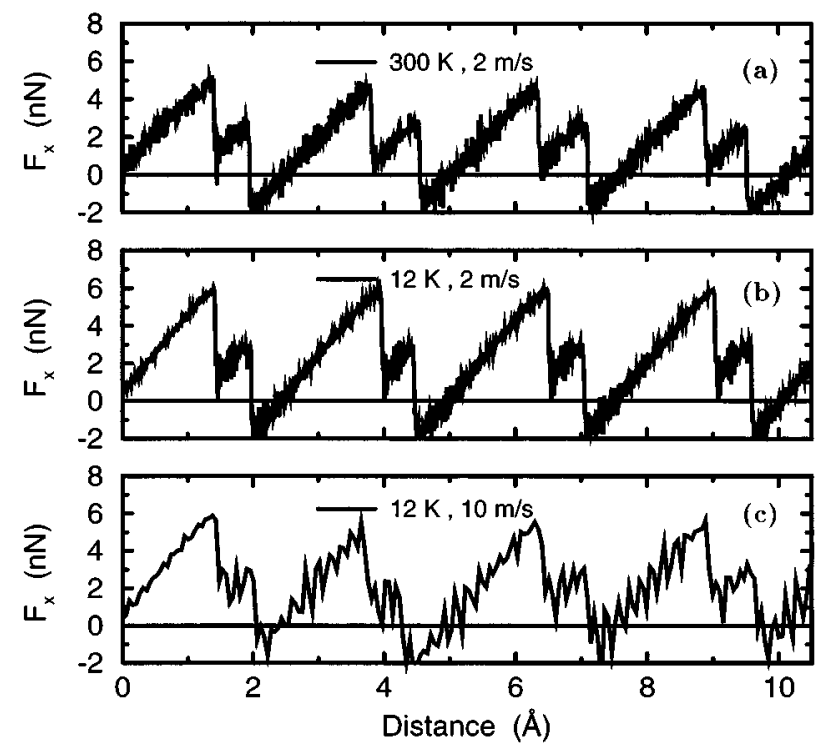

FIG. 9. The lateral force $F_{x}$ on the tip as a function of the sliding distance at different temperatures and sliding velocities. The contacting $\mathrm{Cu}(111)$ surfaces have identical orientation. $T=0 \mathrm{~K}$.

large fluctuations are mainly due to a systematic motion and not only thermal motion. Immediately after a slip, the average kinetic energy per atom is increased by an amount corresponding to about $5 \mathrm{~K}$, but the magnitude of the fluctuations are much too large compared to the fluctuations at 300 $\mathrm{K}$ [Fig. 9(a)] to be due to an increase in temperature. When a slip occurs, phonons are excited, and these phonons are damped as the increase in the kinetic energy is drained out of the system by the temperature control in the top of the tip and in the bottom of the substrate.

From the fluctuations in the $F_{x}$ curves at $12 \mathrm{~K}$, it looks like almost all the released elastic energy is dissipated during the long sticks at $2 \mathrm{~m} / \mathrm{s}$, but at $10 \mathrm{~m} / \mathrm{s}$, this is clearly not the case. In the latter case, the fluctuations increase significantly after the first slip, and they remain large for the rest of the simulation. At all the considered velocities, there is not

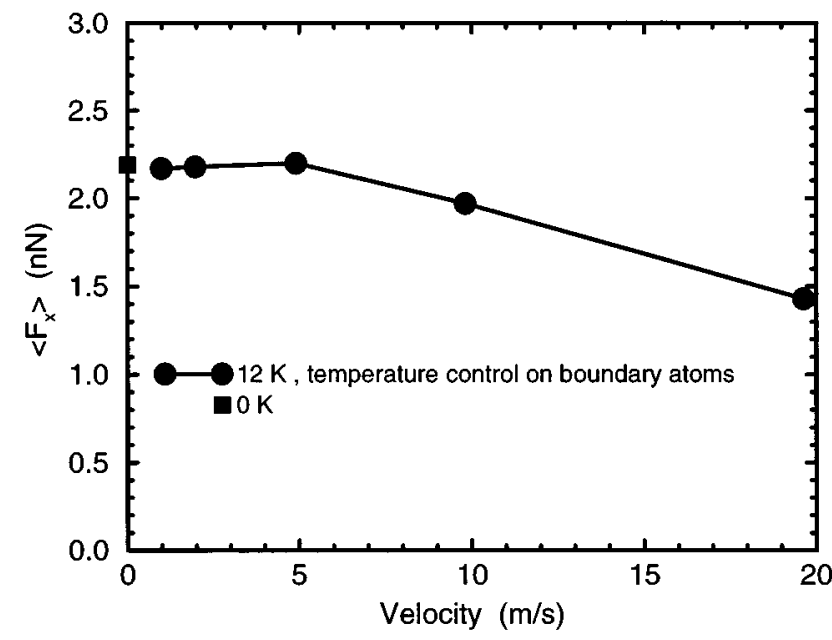

FIG. 10. The average lateral force $\left\langle F_{x}\right\rangle$ on the tip as a function of the sliding velocity. The contacting $\mathrm{Cu}(111)$ surfaces have identical orientation. 
enough time during the short stick for complete energy dissipation to occur. By comparing the two $F_{x}$ curves in Fig. 9(b) and Fig. 9(c), one finds that the slips tend to occur earlier at $10 \mathrm{~m} / \mathrm{s}$ than at $2 \mathrm{~m} / \mathrm{s}$. Also, the maximum values of the lateral force are smaller at the high velocity compared to the low velocity. This indicates that phonons excited at previous slips can promote subsequent slip events. This "phonon mechanism" decreases $\left\langle F_{x}\right\rangle$, as illustrated in Fig. 10. The filled circles in Fig. 10 show $\left\langle F_{x}\right\rangle$ as a function of the sliding velocity. The averages are calculated from the three last periods of the $F_{x}$ curves. For comparison, the filled square represents the result from a simulation at $T=0 \mathrm{~K}$ done with MD minimization.

In the MD simulations, the energy dissipation is determined by the temperature control. We apply the temperature control at the boundary of the system in order to minimize the artificial perturbations of the dynamics of the atoms at the sliding interface. On the other hand, it is possible to choose a very efficient temperature control by simply using Langevin dynamics on all the dynamic atoms in the system. In such simulations, the system behaves "less dynamically" in the sense that the systematic atomic motion, due to the excited phonons, are damped extremely rapidly. Therefore, the "phonon mechanism" is not active. Instead, one finds that $\left\langle F_{x}\right\rangle$ increases with the sliding velocity. This is due to another mechanism by which the sliding velocity affects the frictional properties. The sliding of the tip is induced by displacing the top part of the tip. In the subsequent time steps in the simulations, this perturbation propagates through the tip. Therefore, at higher sliding velocities, the bottom part of the tip is "delayed" compared to the top part of the tip. This "delay mechanism" tends to make the slips take place at a larger displacement at higher velocities than at lower velocities, and this increases $\left\langle F_{x}\right\rangle$. The "delay mechanism" is also present in the simulations with temperature control on the boundary layers (the filled circles in Fig. 10), but in this case, the "phonon mechanism" is more important, so that the net result is a decreasing $\left\langle F_{x}\right\rangle$. At $300 \mathrm{~K}$, phonons are damped more efficiently by the temperature control imposed by the Langevin dynamics at the boundary. Therefore, at $300 \mathrm{~K}$, the "delay mechanism" is more important than the "phonon mechanism" in the relevant range of velocity.

Figure 10 shows that at a temperature of $12 \mathrm{~K}$, MD simulations at velocities in the order of $1-2 \mathrm{~m} / \mathrm{s}$ give the same results as the simulations of quasistatic sliding at $0 \mathrm{~K}$. This also holds if one applies temperature control on all atoms in the system. This indicates that within the time scales that are accessible in MD simulations, there is no effect of the sliding velocity for velocities below $2 \mathrm{~m} / \mathrm{s}$. However, one cannot exclude that thermally activated processes could be important at higher temperatures and on longer time scales.

\section{B. $\mathrm{Cu}(111)$ tip/ $\mathrm{Cu}(111)$ surface, nonmatching surfaces}

As mentioned in the Introduction, both experimental and theoretical studies indicate that commensurability is an important concept in the context of sliding friction between clean atomically flat solid surfaces..$^{23,27,28}$ The possibility has been suggested that two incommensurate surfaces can slide over one another without any friction..$^{23,27,28}$ Some studies predict that this could happen even in metallic systems. ${ }^{28} \mathrm{In}$

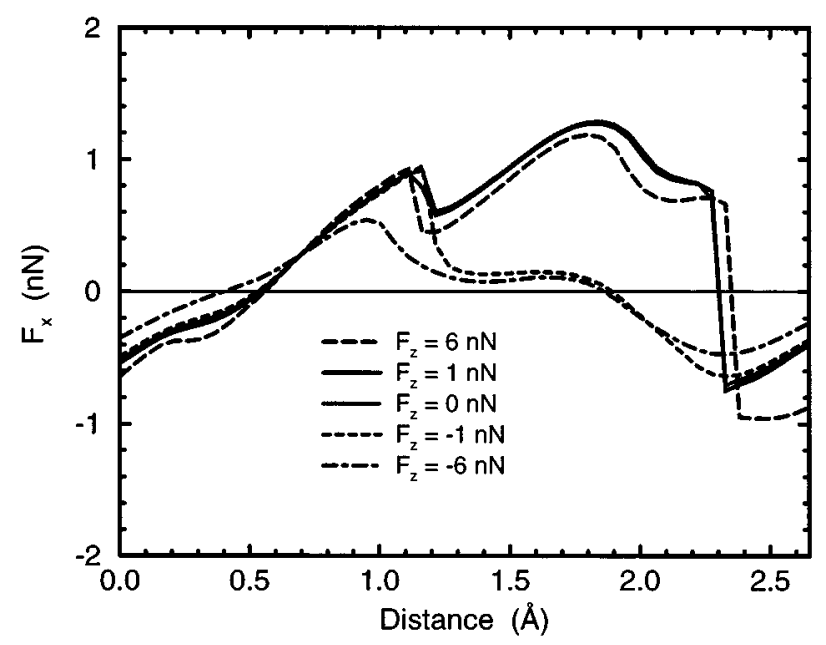

FIG. 11. The lateral force $F_{x}$ on the tip as a function of the sliding distance at different loads $F_{z}$. The misorientation angle of the $\mathrm{Cu}(111)$ surfaces is $\theta=16 \cdot 1^{\circ} . T=0 \mathrm{~K}$.

order to investigate the effect of interfacial mismatch on the frictional properties, a series of simulations have been performed for a system like the one in Fig. 1, except that the $\mathrm{Cu}(111)$ tip is rotated an angle of $16.1^{\circ}$ around an axis perpendicular to the $\mathrm{Cu}(111)$ surface. The sliding direction is the [101] direction of the substrate as in Sec. III A. The simulations are done using MD minimization. The areas of the tip-substrate contacts in the simulations are rather small, and this gives rise to finite size effects, as discussed below.

The simulations have been performed with five different loads among which some are positive and others are negative. The curves in Fig. 11 show the lateral force $F_{x}$ on the tip as a function of the sliding distance. At negative loads, the curves are smooth and the average lateral force $\left\langle F_{x}\right\rangle$ is zero, within the computational accuracy. At positive loads, a kind of stick and slip is observed, and $\left\langle F_{x}\right\rangle$ is positive. When the load is increased, there is a transition from a superlubric state without kinetic friction to a stick and slip state with finite kinetic friction. Because of the mismatch, the stick and slip is not related to the kind of zigzag motion that was discussed in Sec. III A. In the present case, only one slip occurs in each period. It should be mentioned that at the highest load $F_{z}=6.0 \mathrm{nN}$, the $F_{x}$ curve is not periodic. In this particular simulation, the bottom layer of the tip sticks so well to the substrate, that the sliding takes place between the lowest and the second-lowest layer of the tip. The other $F_{x}$ curves are periodic. Compared to the simulations with matching surfaces in Sec. III A, $F_{x, \max }$ is smaller by approximately a factor of five.

Next, we investigate the question of how typical the situation with finite kinetic friction is, and under which conditions it occurs. First, the effect of the contact area is studied. Figure 12 shows $F_{x}$ as a function of the sliding distance in simulations with three different tip sizes. The height of the top of the tip is the same in the three simulations. The average normal pressure $\left\langle p_{z}\right\rangle$ lies in the range from $0.6 \mathrm{GPa}$ at the smallest contact area to $2.0 \mathrm{GPa}$ at the largest area. One finds that the curve corresponding to $9 \times 9$ atoms in the contact is quite similar to the one with $5 \times 5$ atoms. As in some 


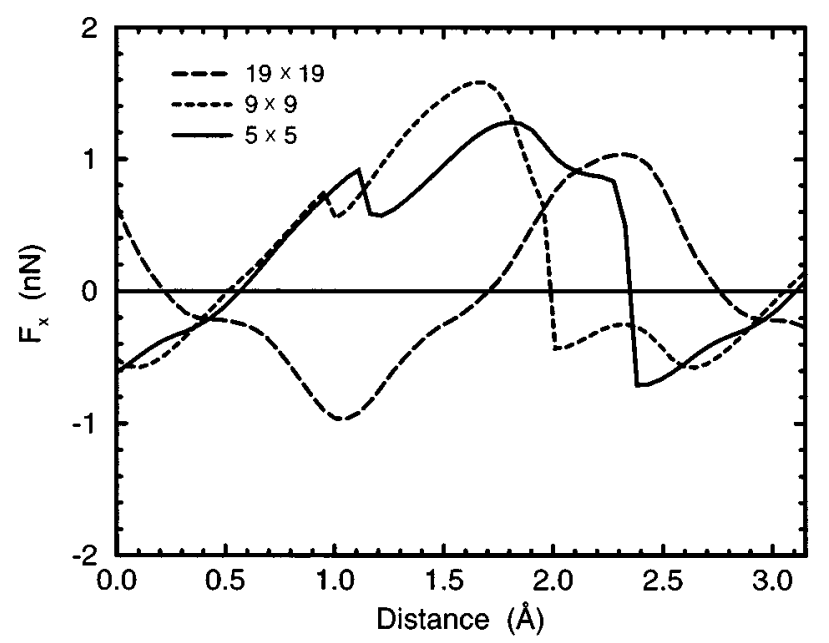

FIG. 12. The lateral force $F_{x}$ on the tip as a function of the sliding distance at three different contact areas. The tip starts out in a position in which the atom in the middle of the bottom layer of the tip is in a perfect fcc position on the surface of the substrate. The misorientation angle of the $\mathrm{Cu}(111)$ surfaces is $\theta=16 \cdot 1^{\circ} . T=0 \mathrm{~K}$.

of the simulations presented in Fig. 11, stick and slip occurs, and $\left\langle F_{x}\right\rangle$ is positive. In the simulation with a contact area of $19 \times 19$ atoms, there is no stick and slip and $\left\langle F_{x}\right\rangle$ is zero, within the computational accuracy. The maximum lateral force $F_{x \text {, max }}$ does not increase significantly with the contact area-at least not proportionally to the area.

In the simulations that have been mentioned so far in Sec. III $\mathrm{B}$, the starting position of the tip has been chosen so that the atom in the middle of the bottom layer of the tip is placed in a perfect fcc position on the surface of the substrate. It turns out that changing the $y$ position of the tip before scanning in the $x$ direction can change the frictional properties drastically when there is a mismatch at the interface. Simulations similar to the ones in Fig. 12 have been done at other starting positions. Figure 13 shows the $F_{x}$ curves in some

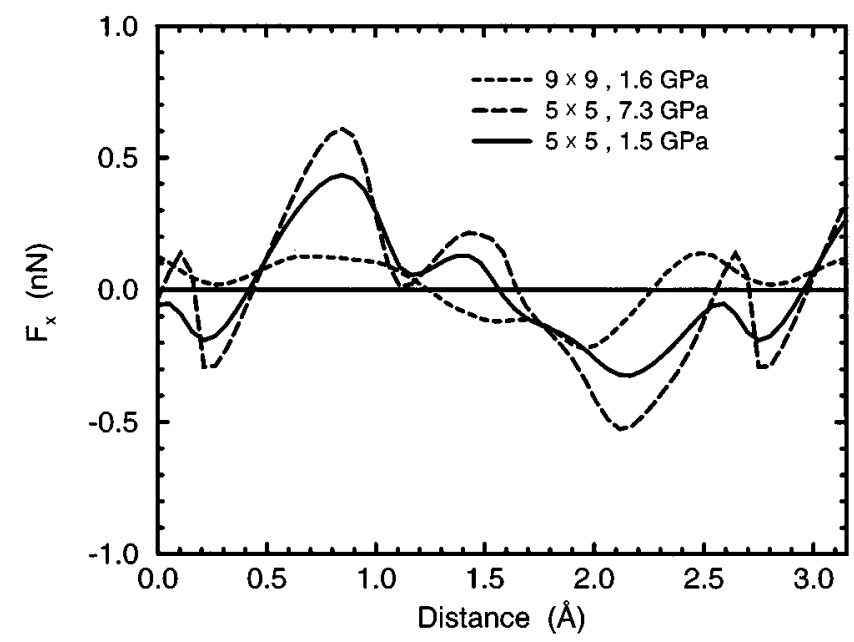

FIG. 13. The lateral force $F_{x}$ on the tip as a function of the sliding distance. The scans are at $y$ positions that are different from the ones in Fig. 12. The misorientation angle of the $\mathrm{Cu}(111)$ surfaces is $\theta=16.1^{\circ} . T=0 \mathrm{~K}$.
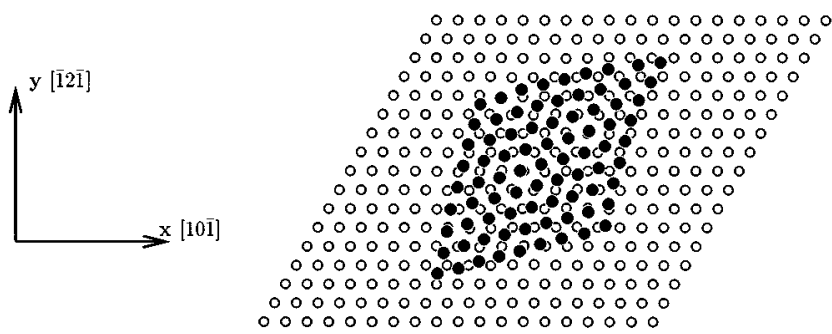

FIG. 14. The bottom $\mathrm{Cu}(111)$ layer of the tip on the top $\mathrm{Cu}(111)$ layer of the substrate, in the configuration with maximum $F_{x}$ for a simulation with a contact area of $9 \times 9$ atoms (short-dashed curve in Fig. 12). The sliding direction is to the left. Note that the upper right corner is pinned to the substrate.

selected cases with a $5 \times 5$ atoms contact and a $9 \times 9$ atoms contact, respectively. In these cases superlubricity occurs (i.e., $\left\langle F_{x}\right\rangle$ is zero), and this holds even if $\left\langle p_{z}\right\rangle$ is increased substantially. The maximum lateral force $F_{x, \max }$ does not increase with the contact area.

In all of these simulations with idealized tip-surface geometries, positive and finite friction $\left(\left\langle F_{x}\right\rangle>0\right)$ is unambiguously connected to stick and slip motion. The studies described in this section show that stick and slip is a fragile property when there is a mismatch at the interface. One can get further insight into the stick and slip phenomenon by looking at the positions of the atoms at the interface during the sliding. As an example, we consider the simulation corresponding to the short-dashed line in Fig. 12. In this simulation with a contact of $9 \times 9$ atoms, stick and slip occurred. Figure 14 shows the bottom layer of the tip and the top layer of the substrate in the configuration with the maximum lateral force $F_{x, \max }$. It is seen that the last row of atoms in the bottom layer of the tip bends backwards. The atoms in the upper right corner are pinned to the substrate. This effect is present in the stick and slip simulations, but not in those simulations that do not exhibit stick and slip (e.g., the one corresponding to the short-dashed curve in Fig. 13).

The overall conclusion regarding sliding friction between misoriented $\mathrm{Cu}(111)$ surfaces is that in the case of small contacts, sliding can give rise to a finite, positive average lateral force $\left\langle F_{x}\right\rangle$. In such cases, this is due to local pinning that under favorable conditions (normal pressure, local matching of atomic positions) can occur at the corners of the interface. At sufficiently large contact areas, this boundary effect becomes unimportant, and, in general, the average lateral force per unit of contact area is vanishing at large contact areas. This will be discussed further in Sec. III D and in Sec. III E.

\section{C. $\mathrm{Cu}(100)$ tip/ $\mathrm{Cu}(100)$ surface, matching surfaces}

This section describes some simulations in which the planes parallel to the surface are the (100) planes. Otherwise, the geometry of the system is similar to the one in Fig. 1. The close-packed $\{111\}$ planes are the preferred slip planes in the fcc metals. Keeping this in mind, it seems reasonable that in Sec. III A, the sliding took place at the interface between the tip and the surface, and wear did not occur. It is not obvious what will happen when it is the (100) planes that are 

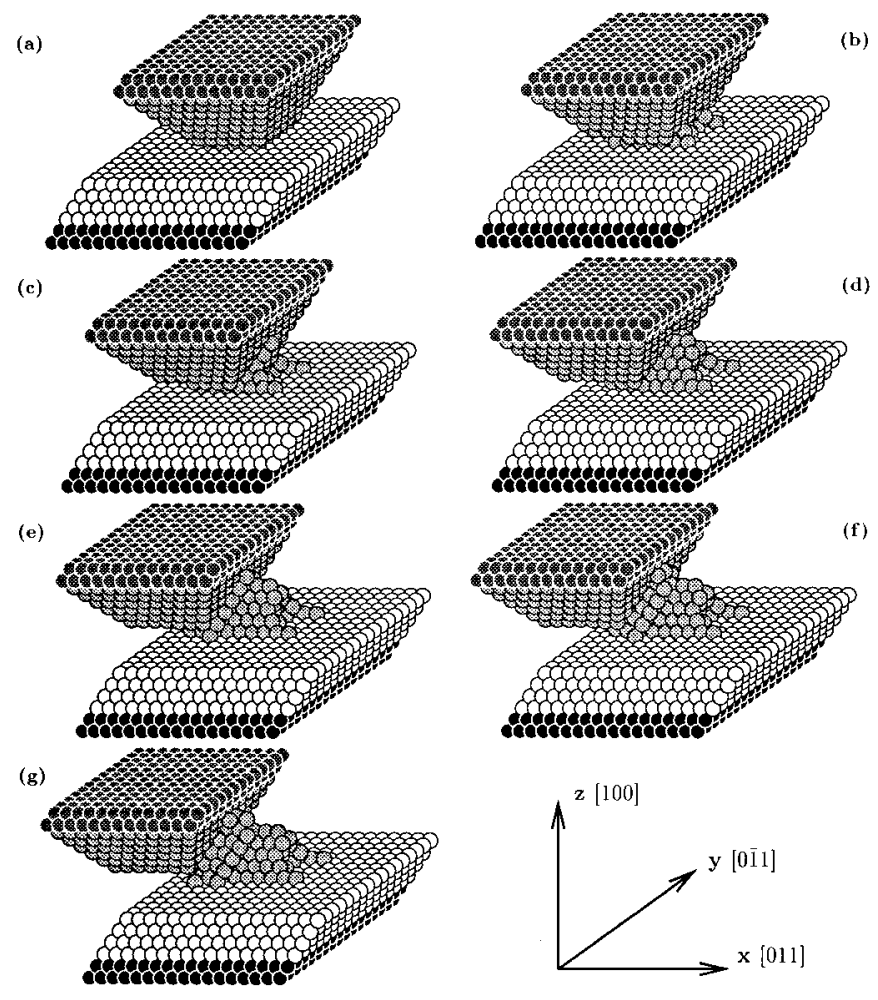

FIG. 15. Snapshots of a $\mathrm{Cu}(100)$ tip on a $\mathrm{Cu}(100)$ substrate during sliding. Picture (a) shows the starting configuration. The other snapshots are taken just after the 2, 4, 6, 8, 10, and the 12 slip, respectively. $T=0 \mathrm{~K}$.

parallel to the surface. On the one hand, the cross-sectional area is smallest at the interface between the tip and the surface. On the other hand, the $\{111\}$ planes are the preferred slip planes in the fcc metals, since the critical shear stress is larger for slip between $\{100\}$ planes than for slip between $\{111\}$ planes.

Two simulations have been done using MD minimization. The tip is scanned in the close-packed [01 1 ] direction of the substrate at a constant height and at a constant load, respectively. The coordinate system is chosen so that the $x$ direction is opposite to the sliding direction, and the $z$ direction is along the [100] direction, perpendicular to the surface. The starting point of the simulations is a configuration in which the load $F_{z}$ is $0.5 \mathrm{nN}$. This load corresponds to a normal pressure $p_{z}$ of $0.3 \mathrm{GPa}$. The results of the constant-height simulation are presented and discussed in details. The constant-load scan is only discussed briefly.

\section{Constant-height scan}

Figure 15 shows some snapshots of the system during the simulation. One finds that, in general, the sliding does not occur at the interface between the tip and the substrate. Instead, the sliding tends to take place between the $\{111\}$ planes inside the tip. During the sliding, material is transferred from the tip to the substrate. In other words, adhesive wear is occurring. The same behavior is seen in MD simulations at $12 \mathrm{~K}$ and at $300 \mathrm{~K}$ with a sliding velocity of $2 \mathrm{~m} / \mathrm{s}$.

Figure 16(a) shows the lateral force $F_{x}$ on the tip as a function of the sliding distance in the constant-height scan.
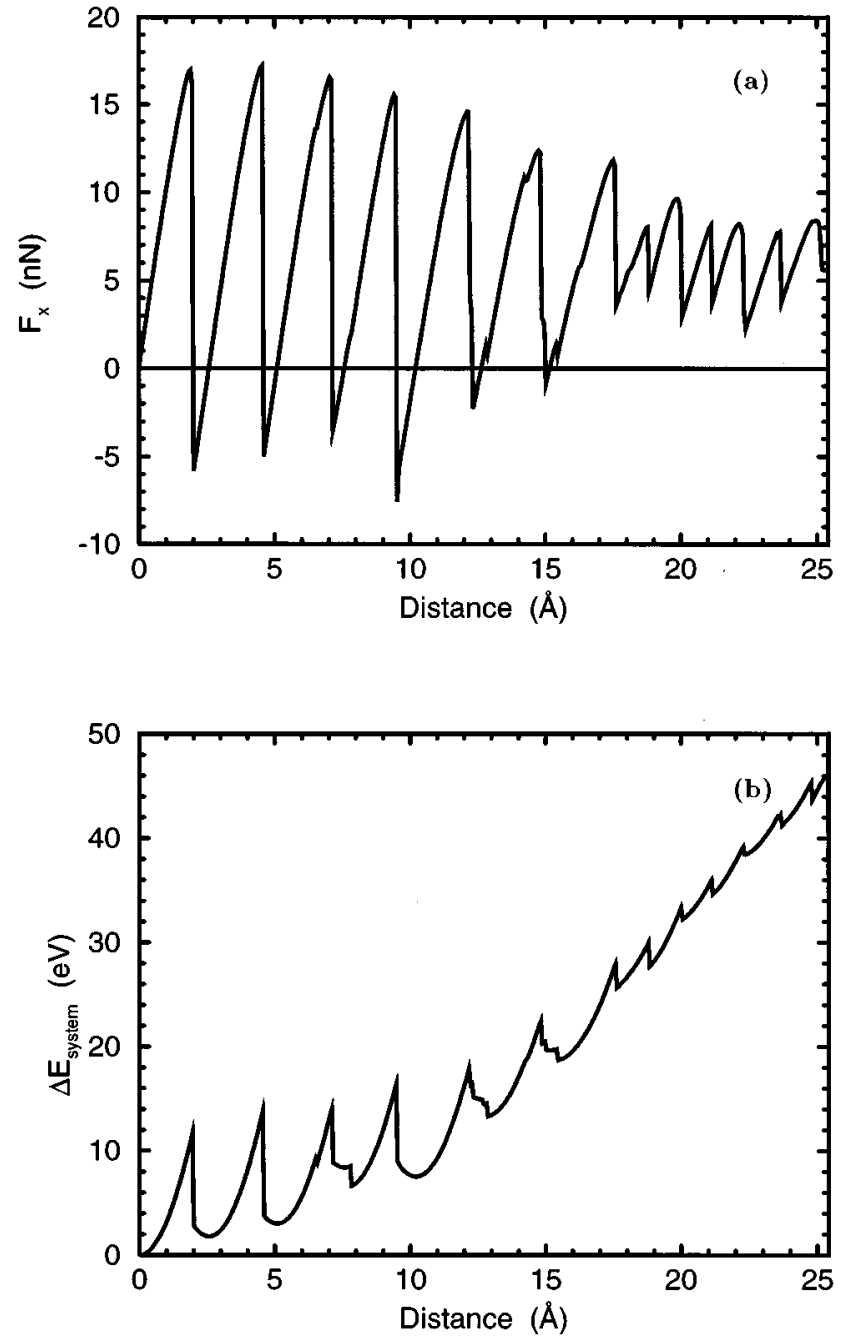

FIG. 16. (a) The lateral force $F_{x}$ on the tip as a function of the sliding distance. (b) The increase of the total energy $\Delta E_{\text {system }}$ of the system, as a function of the sliding distance. The contacting $\mathrm{Cu}(100)$ surfaces have identical orientation. $T=0 \mathrm{~K}$.

The $F_{x}$ curve has the kind of sawtooth shape that is characteristic of stick and slip motion. The curve does not repeat itself periodically, and this is an indication of plastic deformations. The curve can be divided into two parts. In the first part, the distances between subsequent slip events are approximately $2.4-2.7 \AA$. That is, one slip takes place for each period of the substrate. In the last part of the curve, two slips occur for each period, since the distances between slips are 1.2-1.4 $\AA$. During the scan, $F_{z}$ varies between $+6 \mathrm{nN}$ (repulsion) and $-19 \mathrm{nN}$ (attraction).

Quantitatively, in the beginning of the sliding process, both $\left\langle F_{x}\right\rangle$ and the maximum values of $F_{x}$ are larger than the ones in III A by approximately a factor of three. Generally, the local maxima of $F_{x}$ tend to decrease during the sliding. However, the average value of $F_{x}$ in a period decreases only slightly. The small stick and slips, in the last part of the sliding process, are related to the kind of zigzag motion that was discussed in Sec. III A, for sliding between $\{111\}$ planes.

The total energy of the system is shown in Fig. 16(b). The local maxima are due to elastic deformations. The overall increase is caused by plastic deformations. As the sliding 
proceeds, more atoms occupy unfavorable positions (they have a low coordination number), and this increases the energy. In this way another mechanism for transformation of energy is introduced. In the simulation, the increase in the total energy is $46 \mathrm{eV}$. This number should be compared to the total work, which can be calculated as $\left\langle F_{x}\right\rangle$ times the total sliding distance. The result is $103 \mathrm{eV}$. In the simulation, the remaining energy of $57 \mathrm{eV}$ is removed by the minimization algorithm. In a MD simulation, phonons would be excited, and the released elastic energy would go into kinetic energy, which would be removed from the system by the temperature control. The relative importance of the two mechanisms for energy transformation changes during the simulation. In the beginning, the phonon excitations are most important. However, as the loss of coordination number becomes large compared to the number of atoms sliding over each other, the plastic deformation is responsible for most of the energy transformation.

The described deformation mechanism is different from the ones reported by Nieminen et al. from MD simulations of a similar system. ${ }^{32}$ In their simulations, the sliding takes place between $\{100\}$ layers inside the tip. The major differences, of their and our simulations, are that they use Morse pair potentials to describe the interatomic interactions and that the sliding velocity in their simulations is as high as 100 $\mathrm{m} / \mathrm{s}$.

\section{Constant-load scan}

In the constant-load scan, the static atoms in the top of the tip are moved towards and away from the substrate in order to keep the normal force $F_{z}$ constant at $0.5 \mathrm{nN}$ (within an accuracy of $0.1 \mathrm{nN}$ ). The constant-load scan fully confirms that the sliding takes place between the $\{111\}$ planes inside the tip. In fact, it happens right from the first slip. Because of the wear process, the tip is compressed. The local maxima of the $F_{x}$ curve tend to increase during the sliding, in contrast to the constant-height scan.

\section{D. $\mathrm{Cu}(100)$ tip/Cu(100) surface, nonmatching surfaces}

In order to further investigate the effect of interfacial mismatch, two other constant-height scans have been simulated with the $\mathrm{Cu}(100)$ tip after a rotation of $16.3^{\circ}$ and $36.9^{\circ}$, respectively, around an axis perpendicular to the $\mathrm{Cu}(100)$ surface. The position of the atom in the middle of the bottom layer of the tip is unchanged by the rotations. The initial normal load is $0.5 \mathrm{nN}$ and the sliding direction is the closepacked $[0 \overline{1} \overline{1}]$ direction of surface of the substrate.

In the simulation with $\theta=16.3^{\circ}$, the sliding takes place between the two lowest layers of the tip. The pinning effect described in Sec. III B makes the bottom layer stick to the substrate. The maximum value of the lateral force is about one quarter of the value in the case of matching surfaces. At $\theta=36.9^{\circ}$, the tip slides over the substrate without stick and slip and without wear. The average lateral force is zero.

\section{E. $\mathrm{Cu}(\mathbf{1 0 0})$ twist grain boundaries}

So far the studies of the effect of interfacial mismatch have dealt with small contacts with idealized geometries and it seemed like boundary effects were important. To clarify

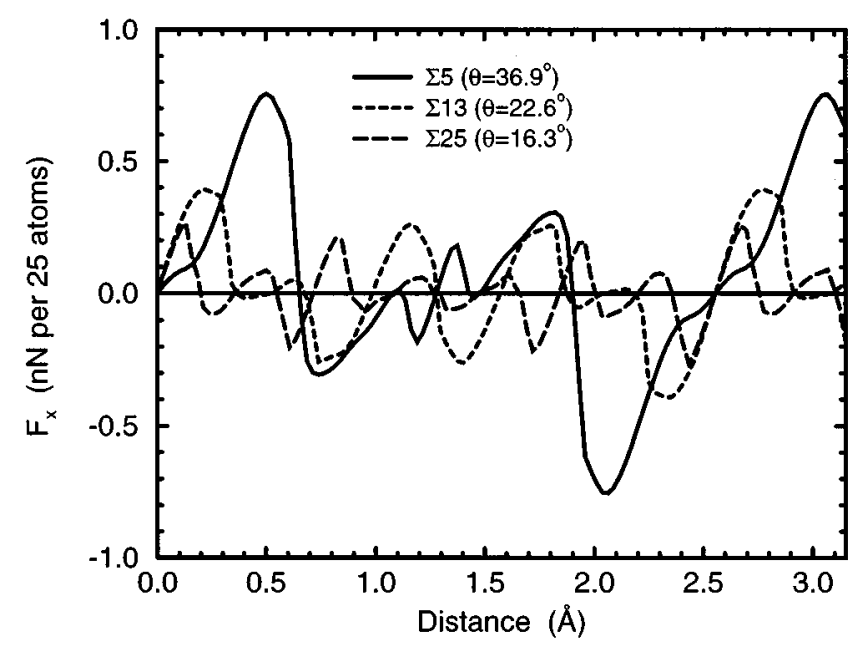

FIG. 17. The lateral force $F_{x}$ on the tip as a function of the sliding distance for three $\mathrm{Cu}(100)$ twist grain boundaries. The coincident site boundaries are $\Sigma 5\left(\theta=36.9^{\circ}\right), \Sigma 13\left(\theta=22.6^{\circ}\right)$, and $\Sigma 25\left(\theta=16.3^{\circ}\right)$, respectively. $T=0 \mathrm{~K}$.

this further, we have studied sliding between two slabs, both exposing $\mathrm{Cu}(100)$ surfaces. The upper slab is rotated an angle $\theta$ around an axis perpendicular to the surface. We then get $\mathrm{Cu}(100)$ twist grain boundaries. Three different coincidence site boundaries have been studied. These are labeled $\Sigma 5\left(\theta=36.9^{\circ}\right), \quad \Sigma 13\left(\theta=22.6^{\circ}\right)$, and $\Sigma 25\left(\theta=16.3^{\circ}\right)$, respectively. The value of $\Sigma$ denotes the inverse of the fraction of coincident sites.

Figure 17 shows the lateral force $F_{x}$ on the upper slab when sliding in the $[0 \overline{1} \overline{1}]$ direction of the surface of the lower slab, in the three simulations. The normal load is approximately zero in all cases. The lateral force is normalized so that it corresponds to a contact area of 25 contact atoms.

One finds that the maximum values of $F_{x}$ are relatively small, and the average values are zero within the computational accuracy. There is no stick and slip motion and no kinetic friction. The fact that no stick and slip and no kinetic friction is observed confirms the suggestion in previous sections that the occurrence of stick and slip is related to finite size and boundary effects.

When $\Sigma$ is large, there are more wiggles on the $F_{x}$ curve. This reflects that the energy-translation surface have more local minima. Furthermore, the maximum value of $F_{x}$ is smaller for boundaries with large values of $\Sigma$.

\section{F. Neck between $\mathrm{Cu}(100)$ surfaces}

Until this point, systems with very idealized geometries have been studied. The tips have been cut along low-indices planes in the fcc structure and in all cases, the smallest cross section have been at the interface between the tip and the flat substrate. This geometry has the advantage of being very well defined.

The idea in this section is to perform simulations that are closer to an experimental situation on the atomic scale, e.g., a scanning tunneling microscope (STM) or atomic force microscope (AFM) experiment. In recent experiments, a contact is established by indentation of a STM/AFM tip into a substrate and the mechanical and transport properties are 
(a)
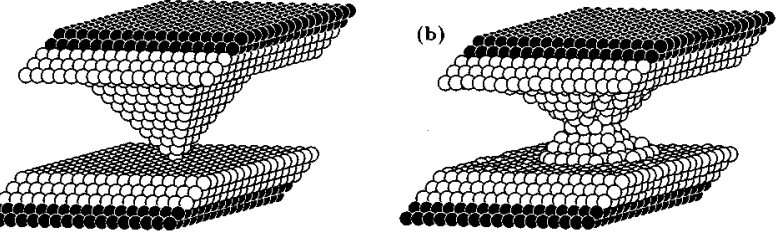

FIG. 18. Forming a neck between two matching $\mathrm{Cu}(100)$ surfaces. (a) The initial configuration. (b) After annealing.

then studied during retraction of the tip. ${ }^{12-18,20}$ On the time scales of a typical experiment at room temperature, diffusion processes can influence the contact between the tip and the substrate. A system like the one in Fig. 1 is not a very stable one, since many atoms occupy unfavorable positions at corners and edges. In this section, a more realistic tip-surface contact is studied.

Because of the separation of time scales, it is not possible to form a stable contact by simulating diffusion in MD simulations at room temperature or below. Instead, we establish a contact in the following way: First, a sharp tip with a shape of a pyramid turned upside down is placed on the upper substrate. The sharp end of the tip is in contact with the lower substrate, as seen in Fig. 18(a). The tip is then heated to a temperature of $1050 \mathrm{~K}$ and annealed for $270 \mathrm{ps}$, using Langevin dynamics. At the high temperature, the mobility of the atoms is high, and atoms migrate to the lower substrate, and a neck forms. The neck is cooled down to about $300 \mathrm{~K}$ and then compressed a little. The result is the neck shown in Fig. 18(b).

The mechanical response of such a neck during stretching has been simulated and discussed in Ref. 12. The neck is seen to undergo a series of mechanical instabilities as suggested in Ref. 30 and the mechanical instabilities are related to emission of dislocations. In the following, we shall study the response of the neck to a shearing motion.

\section{Shearing of a neck}

The shearing of the neck is studied in MD simulations at $300 \mathrm{~K}$ and at $12 \mathrm{~K}$. The temperature is controlled by Lange-

(a)

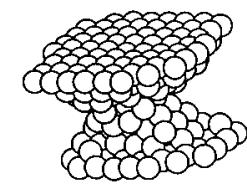

(c)

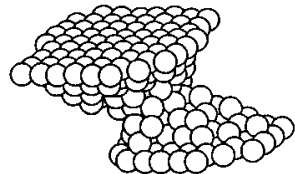

(e)
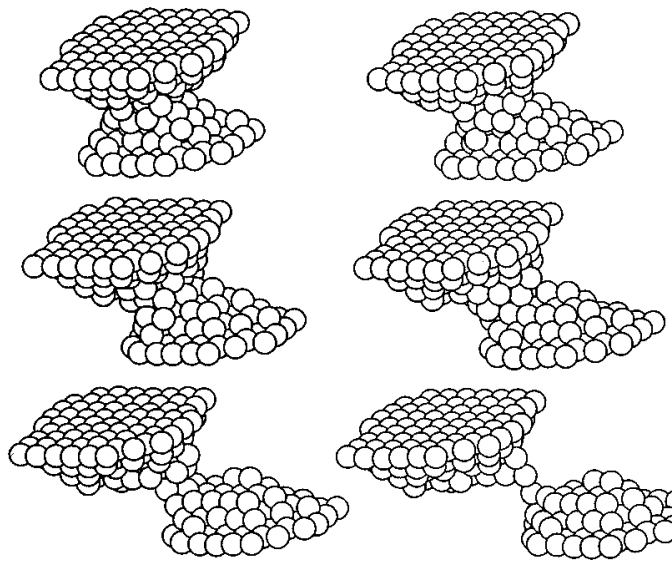

(d)

(f)

FIG. 19. Snapshots of a $\mathrm{Cu}(100)$ neck during shearing. Picture (a) shows the starting configuration. The upper substrate has been displaced $4.2 \AA$ between subsequent pictures. The substrate atoms are not shown in the pictures. $T \approx 300 \mathrm{~K}$.
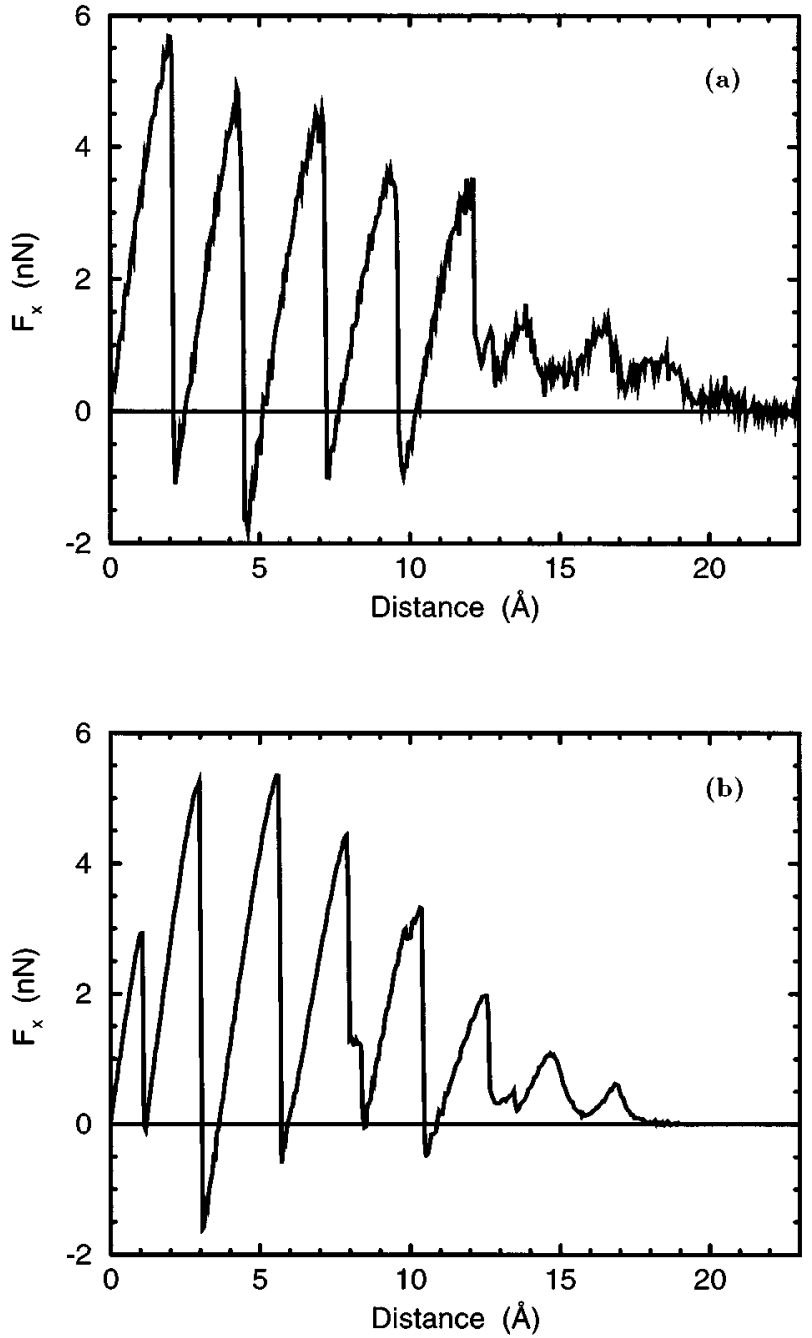

FIG. 20. The lateral force $F_{x}$ on the substrate above a $\mathrm{Cu}(100)$ neck as a function of the sliding distance. (a) $T \approx 300 \mathrm{~K}$. (b) $T \approx 12 \mathrm{~K}$.

vin dynamics in the outermost dynamic layer in each substrate. After equilibration, the upper substrate is moved in the $[0 \overline{1} \overline{1}]$ direction (the negative $x$ direction), with a velocity of $2 \mathrm{~m} / \mathrm{s}$.

Snapshots of the system in the simulations at $300 \mathrm{~K}$ are shown in Fig. 19. In the first part of the simulation, atoms are sliding over each other in a stick and slip type of motion. The deformation proceeds in alternating elastic and plastic stages. Even in this small contact, the yielding events tend to occur by slip between the close-packed $\{111\}$ planes. Later, the neck gets thinner and the neck is elongated until it finally breaks. The last part of the simulation is more like a lateral stretch of a very thin wire rather than shearing a junction. There are only two or three atoms in the smallest cross section and the atoms in this region rearrange themselves rather smoothly. At $12 \mathrm{~K}$, the mobility of the atoms are lower, and the elongation is not as pronounced. After the neck has broken, one could reverse the direction of the scan, and contact would be reestablished.

Figure 20 shows the lateral force $F_{x}$ as a function of the sliding distance in the two simulations. The lateral force is 
calculated as the sum of the $x$ components of the forces on all the atoms in the neck and in the upper substrate. The $F_{x}$ curves can be divided into two parts. The first part of the $F_{x}$ curve has the well-known sawtooth shape, and $F_{x}$ varies between large positive and smaller negative values. This corresponds to the sliding and stick and slip motion. The lateral force $F_{x}$ is built up during the elastic stages, until yielding occurs and the force suddenly drops. The second part of the $F_{x}$ curve has a smoother variation at smaller positive values. This part corresponds to the situation where the produced thin wire is being stretched. At $12 \mathrm{~K}$, the second part of the $F_{x}$ curve is shorter than at $300 \mathrm{~K}$.

If the neck contains a grain boundary caused by a rotation of the upper substrate, stick and slip can still be observed during shearing. However, the stick and slip motion is more irregular. The distances between successive slips are generally not equal to the distance between nearest-neighboring atoms. The local maxima of $F_{x}$ are smaller by approximately $50 \%$ compared to a perfect crystalline neck. The stick and slip behavior can be explained as due to the locking of atoms near the surface of the neck. Because of the geometry and the small size of the neck, such boundary effects are obviously important.

\section{CONCLUSION}

In this paper, a number of case studies of sliding friction in small systems of copper atoms have been described and discussed. An important frictional behavior is atomic-scale stick and slip, since this is what gives rise to kinetic friction (a nonvanishing average lateral force). Atomic-scale stick and slip is observed for sliding between matching surfaces. There is a tendency for slip to occur between the closepacked $\{111\}$ planes. This is also the case for contacts which have $\{100\}$ planes parallel to the surface. A slip preferably happens by nucleation and subsequent motion of a dislocation.
Atomic-scale stick and slip can also be present in cases of small contacts with interfacial mismatch. The simulations indicate that for sliding between large nonmatching atomically flat surfaces, the kinetic friction is vanishing. However, for smaller contacts pinning is seen to occur at the boundaries of the contact regions and this can give rise to stick and slip and kinetic friction.

Another important concept is wear. In most of the simulations of sliding between $\mathrm{Cu}(111)$ surfaces with very idealized tip-surface geometries, wear does not take place. The smallest cross section is at the interface between the tip and the surface, and the interfacial planes are the close-packed planes. The sliding can, therefore, easily take place at this interface.

However, if we go away from the idealized $\{111\}$ geometries, wear is often occurring. This can happen by local pinning for small contacts with interfacial mismatch or if the planes at the tip-surface interface do not have a $\{111\}$ orientation. More importantly for interpreting STM/AFM experiments at room temperature, annealing of a tip-surface contact is seen to lead to the formation of a neck, where the smallest cross section no longer is found right at the flat surface. The shearing of such a neck leads to a combined sliding and stretching behavior exhibiting stick and slip and where the neck finally breaks, leaving material behind at the surface. The elongation process is seen to be enhanced by temperature.

\section{ACKNOWLEDGMENTS}

Center for Atomic-scale Materials Physics is sponsored by the Danish National Research Foundation. Further funding was obtained from the Danish Research Councils through the Center for Nano-Tribology. We gratefully acknowledge discussions with Jakob Schiøtz and Jens K. Nørskov.
${ }^{1} \mathrm{H}$. Deresiewicz, in Approaches to Modelling of Friction and Wear, edited by F.F. Ling and C.H.T. Pan (Springer-Verlag, New York, 1988).

${ }^{2}$ D. Dowson, History of Tribology (Longman, London, 1979).

${ }^{3}$ F.P. Bowden and D. Tabor, The Friction and Lubrication of Solids (Clarendon, Oxford, 1958).

${ }^{4}$ G. Binnig, C.F. Quate, and Ch. Gerber, Phys. Rev. Lett. 56, 930 (1986).

${ }^{5}$ C.M. Mate, G.M. McClelland, R. Erlandsson, and S. Chiang, Phys. Rev. Lett. 59, 1942 (1987).

${ }^{6}$ R. Erlandsson, G. Hadziioannou, C.M. Mate, G.M. McClelland, and S. Chiang, J. Chem. Phys. 89, 5190 (1988).

${ }^{7}$ O. Marti, J. Colchero, and J. Mlynek, Nanotechnology 1, 141 (1990)

${ }^{8}$ S. Fujisawa, Y. Sugawara, S. Ito, S. Mishima, T. Okada, and S. Morita, Nanotechnology 4, 138 (1993).

${ }^{9}$ G.J. Germann, S.R. Cohen, G. Neubauer, G.M. McClelland, and H. Seki, J. Appl. Phys. 73, 1 (1993).

${ }^{10}$ S. Fujisawa, E. Kishi, Y. Sugawara, and S. Morita, Phys. Rev. B 51, 7849 (1995).
${ }^{11}$ S.R. Cohen, G. Neubauer, and G.M. McClelland, J. Vac. Sci. Technol. A 8, 3449 (1990).

${ }^{12}$ M. Brandbyge, J. Schiøtz, M.R. Sørensen, P. Stoltze, K.W. Jacobsen, J.K. Nørskov, L. Olesen, E. Lægsgaard, I. Stensgaard, and F. Besenbacher, Phys. Rev. B 52, 8499 (1995).

${ }^{13}$ L. Olesen, E. Lægsgaard, I. Stensgaard, F. Besenbacher, J. Schiøtz, P. Stoltze, K.W. Jacobsen, and J.K. Nørskov, Phys. Rev. Lett. 72, 2251 (1994).

${ }^{14}$ J.I. Pascual, J. Méndez, J. Gómez-Herrero, A.M. Baró, N. García, U. Landman, W.D. Luedtke, E.N. Bogachek, and H.-P. Cheng, Science 267, 1793 (1995).

${ }^{15}$ J.I. Pascual, J. Méndez, J. Gómez-Herrero, A.M. Baró, and N. García, Phys. Rev. Lett. 71, 1852 (1993).

${ }^{16}$ V.V. Dremov, S.Yu. Shapoval, and E.V. Sukhorukov, Phys. LowDim. Struct. 11/12, 29 (1994).

${ }^{17}$ N. Agraï, J.G. Rodrigo, C. Sirvent, and S. Vieira, Phys. Rev. B 48, 8499 (1993).

${ }^{18}$ N. Agraï, J.G. Rodrigo, and S. Vieira, Phys. Rev. B 47, 12345 (1993).

${ }^{19}$ J.M. Krans, C.J. Muller, I.K. Yanson, Th. C.M. Govaert, R. Hes- 
per, and J.M. van Ruitenbeek, Phys. Rev. B 48, 14721 (1993).

${ }^{20}$ N. Agrait, G. Rubio, and S. Vieira, Phys. Rev. Lett. 74, 3995 (1995).

${ }^{21}$ D.M. Schaefer, A. Patil, R.P. Andres, and R. Reifenberger, Phys. Rev. B 51, 5322 (1995).

${ }^{22}$ M. Hirano, K. Shinjo, R. Kaneko, and Y. Murata, Phys. Rev. Lett. 67, 2642 (1991).

${ }^{23}$ G.M. McClelland, in Adhesion and Friction, edited by M. Grunze and H.J. Kreuzer (Springer-Verlag, Berlin, 1989).

${ }^{24}$ G.A. Tomlinson, Philos. Mag. 7, 905 (1929).

${ }^{25}$ Y.I. Frenkel and T. Kontorova, Zh. Éksp. Teor. Fiz. 8, 1340 (1938)

${ }^{26}$ M. Peyrard and S. Aubry, J. Phys. C 16, 1593 (1983).

${ }^{27}$ K. Shinjo and M. Hirano, Surf. Sci. 283, 473 (1993).

${ }^{28}$ M. Hirano and K. Shinjo, Phys. Rev. B 41, 11837 (1990).

${ }^{29}$ U. Landman, W.D. Luedtke, and M.W. Ribarsky, J. Vac. Sci. Technol. A 7, 2829 (1989).

${ }^{30}$ U. Landman, W.D. Luedtke, and E.M. Ringer, Wear 153, 3 (1992).

${ }^{31}$ J.A. Harrison, C.T. White, R.J. Colton, and D.W. Brenner, Phys. Rev. B 46, 9700 (1992).

${ }^{32}$ J.A. Nieminen, A.P. Sutton, and J.B. Pethica, Acta Metall. Mater. 40, 2503 (1992).

${ }^{33}$ K.W. Jacobsen, J.K. Nørskov, and M.J. Puska, Phys. Rev. B 35, 7423 (1987).

${ }^{34}$ K.W. Jacobsen, Comments Condens. Matter Phys. 14, 129 (1988).
${ }^{35}$ P. Stoltze, J. Phys. Condens. Matter 6, 9495 (1994), and references therein.

${ }^{36}$ J. Schiøtz, K.W. Jacobsen, and O.H. Nielsen, Philos. Mag. Lett. 72, 245 (1995).

${ }^{37}$ J. Schiøtz, Ph.D. thesis, Physics Department, Technical University of Denmark, Lyngby, Denmark, 1995.

${ }^{38}$ The minimization algorithm is a modification of the usual molecular dynamics algorithm. In each step, the following happens: First, an ordinary molecular dynamics step is performed, i.e., the atomic positions and velocities are updated according to Newtons equation of motion. Second, for each atom, the dot product of the momentum of the atom and the force on the atom $\boldsymbol{p} \cdot \boldsymbol{F}$ is tested. If $\boldsymbol{p} \cdot \boldsymbol{F}<0$, then the momentum is zeroed. Otherwise, the momentum is left unchanged. This procedure brings the system to a local minimum of the total energy.

${ }^{39}$ Fundamentals of Friction: Macroscopic and Microscopic Processes, Vol. 220 of NATO Advanced Study Institute, Series E: Applied Sciences, edited by I.L. Singer and H.M. Pollock (Kluwer, Dordrecht, 1992).

${ }^{40}$ B.J. Briscoe and D.C.B. Evans, Proc. R. Soc. London Ser. A 380, 389 (1982).

${ }^{41}$ A. Kelly, Strong Solids (Clarendon, Oxford, 1973).

${ }^{42}$ The stress tensor is denoted by $\boldsymbol{\sigma}$. The hydrostatic pressure $p$ is defined as $p=-\frac{1}{3} \operatorname{Tr}(\boldsymbol{\sigma})$. The stress deviator $\boldsymbol{\Gamma}$ is given by subtracting $p$ in the diagonal of $\boldsymbol{\sigma}$, i.e., $\Gamma=\boldsymbol{\sigma}-p \boldsymbol{I}$. The second invariant of the stress deviator $J_{2}=\frac{1}{2} \operatorname{Tr}\left(\boldsymbol{\Gamma} \cdot \boldsymbol{\Gamma}^{T}\right)$ is used in von Mises shear stress criterion for plastic yielding. See, e.g., Ref. 30 , and references therein. 